\title{
Interfacial Behaviour of Trilaurylamine as Studied by Electrochemical Methods and Langmuir Films
}

\author{
Hege Ebeltoft, ${ }^{a}$ Kyösti Kontturi, ${ }^{\mathrm{b}, *}$ Lasse Murtomäki ${ }^{\mathrm{b}}$ and Johan Sjöblom ${ }^{\mathrm{a}}$ \\ a Department of Chemistry, University of Bergen, N-5007 Bergen, Norway and baboratory of Physical Chemistry and \\ Electrochemistry, Helsinki University of Technology, Kemistintie 1A, SF-02150 Espoo, Finland
}

\author{
Ebeltoft, H., Kontturi, K., Murtomäki, L. and Sjöblom, J., 1993. Interfacial \\ Behaviour of Trilaurylamine as Studied by Electrochemical Methods and Langmuir \\ Films. - Acta Chem. Scand. 47: 1138-1140. (C) Acta Chemica Scandinavica 1993.
}

Electrochemistry of ion transfer across the aqueous/ organic solution interface has been widely studied during the last decade owing to its importance, e.g. in extraction processes, analytical chemistry (sensors, ion-selective electrodes) and in research into biological membranes. ${ }^{1}$ The selective removal of metal cations or protons from waste waters can be carried out by adding a particular complex former into the organic phase which reacts with the target ion at the interface. The thermodynamics and kinetics of such processes have been studied by several authors, see e.g. Refs. 2-5.

Amines are commonly used as extracting agents for acids, ${ }^{6}$ and recently an electrochemical method was presented showing how the amine-proton stability constants in the organic phase can be evaluated. ${ }^{7}$ In the study, 1,2-dichloroethane was used as an organic solvent owing to its low relative permittivity (10.23), and lauryl (dodecyl), dilauryl and trilauryl amines were used as extracting agents. After submitting this, Matsuda et al. further presented a rigorous theoretical model characterizing the equilibria at the interface during the ion transfer facilitated by neutral macrocyclic ligands. ${ }^{8}$ Their results gave an alternative explanation for the behaviour seen in Fig. 1, i.e. according to Matsuda's results, applied to the case of amines as ligands, the independence of the half-wave potential on $\mathrm{pH}($ when $\mathrm{pH} \leqslant 4$ ) can be explained by the solubility of the amine hydrochloride, while in Ref. 7 this behaviour was assumed to be due to $\mathrm{Cl}^{-}$transfer. Also, experimental evidence was found to support the argument of chloride ion transfer. In order to give a background to Ref. 8 , we briefly show the main issues of the derivation.

\footnotetext{
* To whom correspondence should be addressed.
}

\section{Theoretical}

Consider proton transfer across the aqueous/organic solution interface in the system mentioned above. ${ }^{7}$ This is given by reaction (I), where $A$ denotes amine. For

$\mathrm{H}^{+}(\mathrm{w})+\mathrm{A}(\mathrm{o}) \rightleftharpoons \mathrm{AH}^{+}(\mathrm{o})$

simplicity, the ion pairing of $\mathrm{AH}^{+}(\mathrm{o})$ with the base electrolyte of the organic phase has been omitted. Because

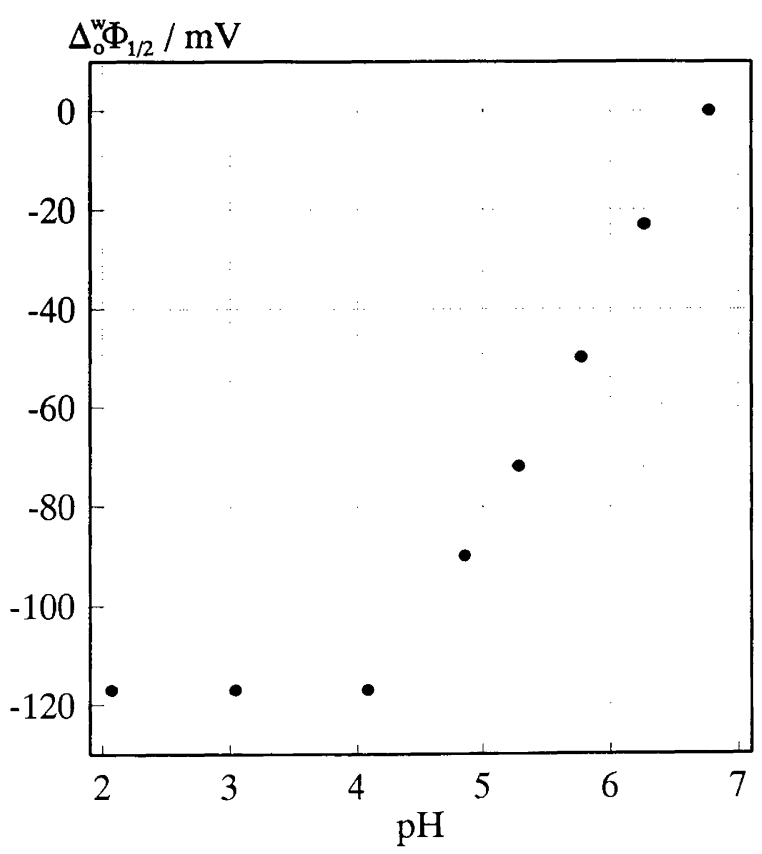

Fig. 1. Polarographic half-wave potentials as a function of $\mathrm{pH}$ for the system of TLA (trilaurylamine) in 1,2-dichloroethane in contact with an aqueous solution; for details see Ref. 7. 
$\mathrm{H}^{+}$is the potential determining ion, the Galvani potential difference across the interface is given by eqn. (1) where

$\Delta_{\mathrm{o}}^{\mathrm{w}} \Phi=\Phi^{\mathrm{w}}-\Phi^{\mathrm{o}}=\Delta_{\mathrm{o}}^{\mathrm{w}} \Phi_{\mathrm{H}^{+}}^{0}+\frac{R T}{F} \ln \frac{a_{\mathrm{H}^{+}}^{\mathrm{o}^{*}}}{a_{\mathrm{H}^{+}}^{\mathrm{w} *}}$

the asterisk denotes interfacial activities. The stability constant of $\mathrm{AH}^{+}(\mathrm{o})$ is given by eqn. (2). Because the

$K_{\mathrm{s}}=\frac{a_{\mathrm{AH}^{+}}^{\mathrm{o}}}{a_{\mathrm{A}}^{\mathrm{o}} a_{\mathrm{H}^{+}}^{\mathrm{o}}}$

diffusion of the amine species limits the current, the polarographic half-wave potential is given by eqn. (3). According to eqn. (3) the half-wave potential is shifted

$$
\begin{aligned}
\Delta_{\mathrm{o}}^{\mathrm{w}} \Phi_{1 / 2}= & \Delta_{\mathrm{o}}^{\mathrm{w}} \Phi_{\mathrm{H}^{+}}^{0} \\
& +\frac{2.3 R T}{F}\left(\mathrm{pH}+\mathrm{p} K_{\mathrm{s}}\right)+\frac{R T}{F} \ln \frac{\gamma_{\mathrm{AH}^{+}}^{\mathrm{o}}}{\gamma_{\mathrm{A}}^{\mathrm{o}}} \\
& \ln \left(\frac{D_{\mathrm{A}}^{\mathrm{o}}}{D_{\mathrm{AH}^{+}}^{\mathrm{o}}}\right)^{1 / 2}
\end{aligned}
$$

ca. $60 \mathrm{mV}$ per $\mathrm{pH}$ unit, and this is seen in several papers, including Ref. 7 at $\mathrm{pH}>4$. This kind of behaviour is described in the paper by Matsuda et al. ${ }^{8}$ by inequality (4), where $K_{\mathrm{s}}^{\mathrm{w}}$ corresponds to eqn. (2) but in the aqueous

$1+\xi_{\mathrm{A}} P_{\mathrm{A}} \gg 20 c_{\mathrm{H}^{+}}^{\mathrm{w}} K_{\mathrm{s}}^{\mathrm{w}}=20 \frac{c_{\mathrm{AH}^{+}}^{\mathrm{w}}}{c_{\mathrm{A}}^{\mathbf{w}}}$

$\xi_{\mathrm{A}}=\left(\frac{D_{\mathrm{A}}^{\mathrm{o}}}{D_{\mathrm{A}}^{\mathrm{w}}}\right)^{1 / 2}$

$P_{\mathrm{A}}=\frac{c_{\mathrm{A}}^{\mathrm{o} *}}{c_{\mathrm{A}}^{\mathrm{w} *}}$

phase. In the experiments of Ref. 7, at pH 2-4 a constant value for the half-wave potential was obtained, which was explained by a competitive transfer of $\mathrm{Cl}^{-}$. In Matsuda's paper this kind of behaviour is seen if inequality (5) holds.

$c_{\mathrm{H}^{+}}^{\mathrm{w}} K_{\mathrm{s}}^{\mathrm{w}}=\frac{c_{\mathrm{AH}^{+}}^{\mathrm{w}}}{c_{\mathrm{A}}^{\mathrm{w}}} \gg 20\left(1+\xi_{\mathrm{A}} P_{\mathrm{A}}\right)$

It is clear that $P_{\mathrm{A}} \gg 1$, and noticing that $K_{\mathrm{s}}^{\mathrm{w}}$ is a thermodynamic constant which applies also at the interface, we obtain from inequality (5) inequality $(6)$, in which $\Gamma$

$1 \gg 20 \xi_{\mathrm{A}} \frac{c_{\mathrm{A}}^{\mathrm{o} *}}{c_{\mathrm{AH}^{+}}^{\mathrm{w} *}} \Gamma$

includes all the activity coefficients, and it is assumed that the surface concentration of $\mathrm{H}^{+}$is equal to its bulk concentration. The latter assumption is certainly valid, since the aqueous side of the interface is practically unpolarized owing to the very high mobility of the proton in water. In order for inequality (6) to be valid in the case of amines as ligands, $\mathrm{AH}^{+}$should be substantially soluble in water in the low $\mathrm{pH}$ range. This is very unlikely because amines are commonly used as extractive agents in industrial processes. In this communication we report on Langmuir films of TLA on aqueous subphases. By preparing a
Langmuir film of the amine one can easily monitor a possible solubility of the film material. Thus when changing the $\mathrm{pH}$ of the solution on which the film is spread we can find out if the film material is dissolved into the subphase or not. In the case of an increased solubility the Langmuir film will completely collapse.

\section{Experimental}

The experiments were performed with a KSV 5000 Langmuir trough double-barrier system (KSV Chemicals Helsinki, Finland). The trough was made of Teflon and the barriers of Delrin. All measurements were made at $21.0 \pm 0.5^{\circ} \mathrm{C}$.

Tridodecylamine ( $>99 \%$ purity, Fluka) was used as supplied. The water used was first purified by distillation. The final purification was undertaken by a Seral pro 90 water purification system (Seral) which included five filter stages: an activated charcoal column, two ion-exchange columns, an absorbent resin column, and a $2 \mu \mathrm{m}$ sterile filter. The $\mathrm{pH}$ of the subphase solution was adjusted by adding $\mathrm{HCl}$ or $\mathrm{NaOH}$ (Suprapur, Merck) to the purified water. The spreading solution was redistilled toluene from Merck.

Surface pressure vs. area isotherms were recorded with a barrier speed of $4 \mathrm{~mm} \mathrm{~min}^{-1}$ and a maximal increase in the surface pressure of $1 \mathrm{mN} \mathrm{m}^{-1} \mathrm{~min}^{-1}$. The quality of the subphase was tested before each run by compressing the surface without any additive. The rise in the surface pressure was always less than $0.05 \mathrm{mN} \mathrm{m}^{-1}$. Isotherms were measured on newly spread monolayers. 30 minutes were allowed for the spreading solvent to evaporate and molecules to rearrange.

For the area relaxation measurements, the film was compressed at a rate of $4 \mathrm{~mm} \mathrm{~min}^{-1}$ with a maximal increase in the surface pressure of $1 \mathrm{mN} \mathrm{m}^{-1} \mathrm{~min}^{-1}$, and then automatically maintained at the desired surface pressure. The change in the surface area was recorded as a function of time.

\section{Surface pressure-area isotherms}

The results obtained are presented in Fig. 2. On pure water ( $\mathrm{pH}$ 5.6) TLA forms slightly expanded films. The film is 'highly' compressible, and the surface area for film collapse is hard to observe. The reason for this is most likely that the surface film starts to bend and fold into multilayers when no longer compressible. The values of the surface pressure at these small areas will exceed the surface tension of pure water, which indicates that one no longer has an ideal contact between water and a TLA monolayer.

When the TLA film is spread upon an alkaline solution $(\mathrm{pH} 11)$ the film is not ionized. The small contraction of the film observed at $\mathrm{pH} 11$, compared to the film at $\mathrm{pH}$ 5.6 , indicates a slight ionization at lower $\mathrm{pH}$ values. As a consequence, the film expands further when the $\mathrm{pH}$ in the subphase is reduced to 1 . In contrast to $\mathrm{pH} 11$ and 5.6 a clear collapse point is now observed at $81 \mathrm{mN} \mathrm{m}^{-1}$. This 


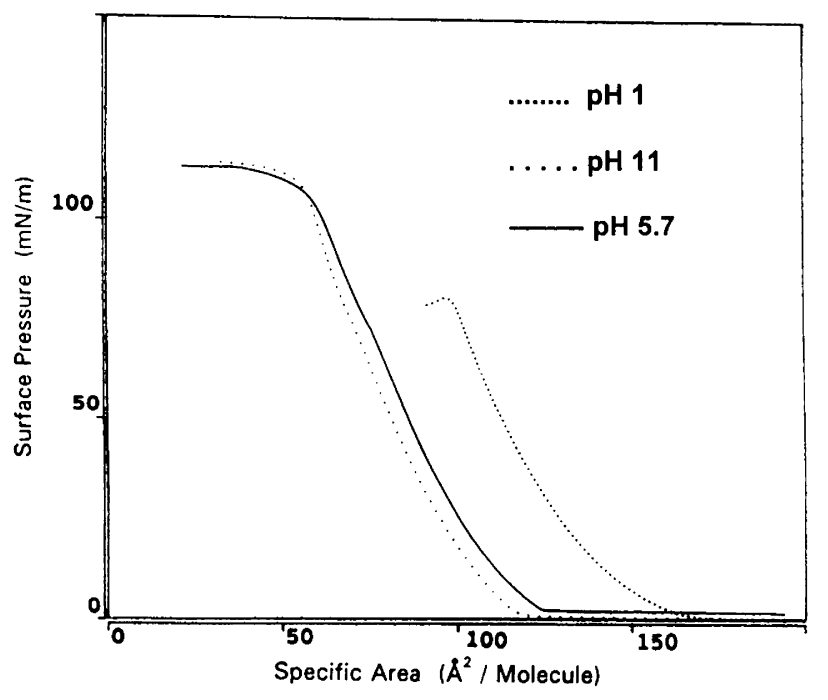

Fig. 2. $n-A$ isotherms of TLA (trilaurylamine) on aqueous subphases with $\mathrm{pH} 1.5,5.7$ and $11 . T=294.0 \pm 0.5 \mathrm{~K}$.

is most likely due to the reduction of the ability of the monolayer to bend and fold upon increased surface charge density.

\section{Relaxation measurements}

When an insoluble monolayer is kept at a constant surface pressure it is often observed that the surface area decreases with time..$^{9-14}$ This is most likely due to one of the following mechanisms: (i) rearrangements of molecules, ${ }^{9,14}$ (ii) dissolution into the subphase ${ }^{14}$ or (iii) transformation to a stable bulk phase. ${ }^{9-13}$ Relaxation due to rearrangement of molecules in the film is characterized by an initial rapid area loss, often depending on the rate of compression, followed by a slower relaxation which asymptotically reaches a constant value. If the film dissolves slowly into the subphase one expects a

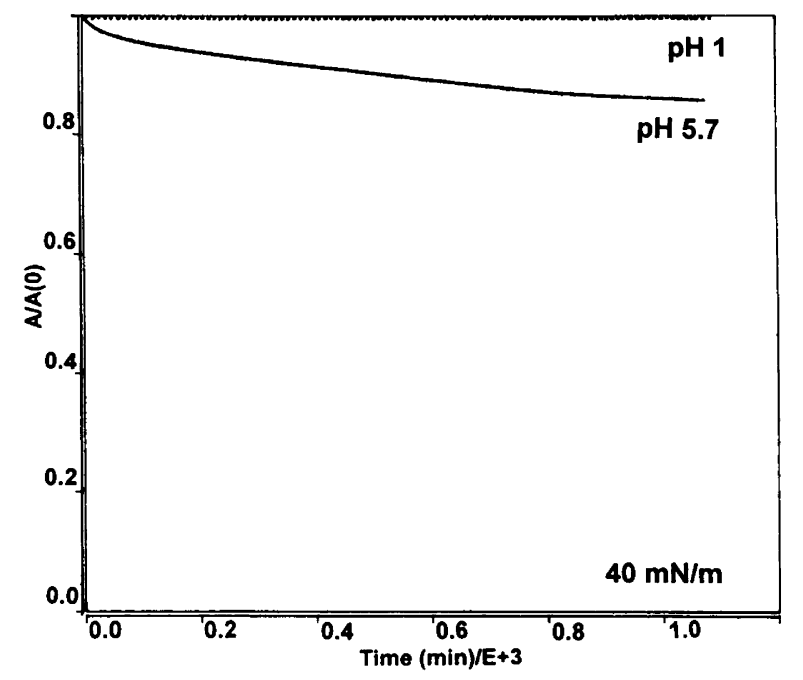

Fig. 3. Monolayer relaxation of TLA (trilaurylamine) on distilled water (pH 5.7) and on aqueous subphase with $\mathrm{pH} 1$ at $40 \mathrm{mN} \mathrm{m}^{-1} . T=294.0 \pm 0.5 \mathrm{~K}$. continuously decreasing area at constant pressure. Nucleation is characterized by a relaxation rate that increases with time.

The results obtained are presented in Fig. 3. Constant surface pressure relaxation measurements of trilaurylamine on pure water at $40 \mathrm{mN} \mathrm{m}^{-1}$ show that after the rearrangements the monolayer slowly relax towards a constant value. At $\mathrm{pH} 1$ and at a constant surface pressure of $40 \mathrm{~m} \mathrm{~N} \mathrm{~m}^{-1}$, the monolayer is completely stable. A relaxation behaviour typical of dissolution processes cannot be observed for the TLA films studied here.

\section{Concluding remarks}

In this study we have investigated Langmuir films of trilaurylamine (TLA) when spread on aqueous subphases. It is found that TLA forms stable monolayers at all $\mathrm{pH}$ values studied ( $\mathrm{pH} 1-11$ ). No dissolution of the fatty amine into the aqueous subphase could be detected. Constant surface pressure relaxation measurements reveal molecular rearrangements in the TLA films, but no dissolution into the subphase. All these data support the explanation presented in Ref. 7, i.e. that a transfer of chloride ions takes place, instead of a dissolution of TLA.

Acknowledgments. Hege Ebeltoft thanks the Norwegian Research Council for Science and the Humanities (NAVF) for a $d r$. scient grant. The Langmuir-Blodget equipment was also financed by NAVF. Johan Sjöblom acknowledges financial support from the Neste Foundation, Helsinki.

\section{References}

1. Girault, H. H. J. and Schiffrin, D. J. In: Bard, A. J. (ed.), Electroanalytical Chemistry, Marcel Dekker, New York 1989, Vol. 15, Chap. 1.

2. Homolka, D., Hung, L. Q., Hofmanová, A., Khalil, M. W., Koryta, J., Mareček, V., Samec, Z., Sen, S. K., Vanýsek, P., Weber, J. and Březina, M. Anal. Chem. 52 (1980) 1606.

3. Wang, E. and Sun, Z. J. Electroanal. Chem. 220 (1987) 235.

4. Koryta, J. J. Electroanal. Chem. 213 (1986) 323.

5. Koryta, J. and Skalický, M. J. Electroanal. Chem. 229 (1987) 265.

6. Marcus, Y. and Kertes, A. S. Ion Exchange and Solvent Extraction of Metal Complexes, Wiley-Interscience, Bristol 1969.

7. Kontturi, A. K., Kontturi, K., Murtomäki, L. and Schiffrin, D. J. Acta Chem. Scand. 46 (1992) 47.

8. Matsuda, H., Yamada, Y., Kanamori, K., Kudo, Y. and Takeda, Y. Bull. Chem. Soc. Jpn. 64 (1991) 1497.

9. Smith, R. D. and Berg, J. C. J. Colloid Interface Sci. 74 (1980) 273.

10. Pezron, E., Claesson, P. M., Berg, J. M. and Vollard, D. J. Colloid Interface Sci. 138 (1990) 245.

11. Neuman, R. D. J. Colloid Interface Sci. 56 (1976) 505.

12. Xu, S., Miyano, K. and Abraham, B. M. J. Colloid Interface Sci. 89 (1982) 581.

13. Honig, E. P., Hengst, J. H. and den Engelsen, D. J. J. Colloid Interface Sci. 45 (1973) 92.

14. Ter-Minassian-Saraga, L. J. Colloid Interface Sci. 11 (1956) 398.

Received April 4, 1993. 